\title{
MORTE ENCEFÁLICA: ASSISTÊNCIA DE ENFERMAGEM
}

\author{
Brain death: nursing care
}

\author{
Cátia Millene Dell Agnoloํ, Rosane Almeida de Freitas ${ }^{1}$, Diogo Fraxino de Almeida', Vanessa Paula Lanjoni², Magda Lúcia \\ Félix de Oliveira ${ }^{3}$.
}

\section{RESUMO}

Morte encefálica representa a parada irreversível das funções encefálicas, e, quando constatada, possibilita ao familiar responsável pelo paciente a doação de órgãos e tecidos para fins de transplante. A manutenção hemodinâmica é essencial para a viabilidade da doação, sendo necessária assistência médica e de enfermagem adequadas. Objetivo: descrever a determinação da ME e a assistência de enfermagem na manutenção do potencial doador de órgãos, através de uma abordagem teórica sobre o assunto. Método: Trata-se de uma pesquisa bibliográfica realizada através de levantamento de artigos, resoluções e manuais relacionados ao tema, em base de dados indexada. Resultados: O potencial doador é todo paciente com lesão encefálica grave, em coma Glasgow 3, de causa conhecida e sem contra-indicações para determinação de ME, submetido a dois exames clínicos e um exame complementar para confirmação. Cabe a toda a equipe de enfermagem conhecer as complicações clínicas que ocorrem na ME e tratar alterações visando a manutenção da viabilidade dos órgãos até o final de todo o processo. Conclusão: A ME envolve aspectos éticos e legais durante sua determinação até a decisão familiar sobre doação. A manutenção da viabilidade dos órgãos deve ser um dos principais objetivos da assistência médica e de enfermagem a potenciais doadores.

Descritores: Morte encefálica, Cuidados de Enfermagem, Transplantes.

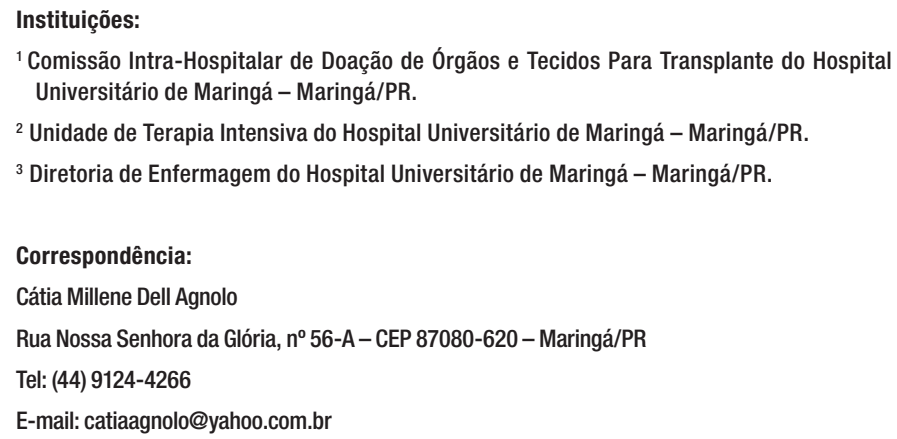

${ }^{1}$ Comissão Intra-Hospitalar de Doação de Órgãos e Tecidos Para Transplante do Hospital Universitário de Maringá - Maringá/PR.

${ }^{2}$ Unidade de Terapia Intensiva do Hospital Universitário de Maringá - Maringá/PR.

${ }^{3}$ Diretoria de Enfermagem do Hospital Universitário de Maringá - Maringá/PR.

Correspondência:

Cátia Millene Dell Agnolo

Rua Nossa Senhora da Glória, n 56-A - CEP 87080-620 - Maringá/PR

Tel: (44) 9124-4266

E-mail: catiaagnolo@yahoo.com.br

Recebido em: 19.10.2009

\section{INTRODUÇÃO}

O diagnóstico de morte encefálica (ME) está longe de ser uniforme, variando conforme o país em que é realizado. ${ }^{1} \mathrm{O}$ Brasil segue o que determina o Conselho Federal de Medicina por meio da resolução ${ }^{\circ}$ 1.480/97, a qual define que a parada total e irreversível das funções encefálicas equivale a morte, conforme critérios estabelecidos pela comunidade cientifica mundial. ${ }^{2}$

Um único doador pode beneficiar mais de 10 pessoas através do transplante de diversos órgãos e tecidos, devendo, portanto, receber a mesma assistência que qualquer paciente de terapia intensiva, pois, para que todos os órgãos sejam viáveis para doação é necessário que estejam bem preservados no momento de retirada ao final de todo o processo. ${ }^{3,4}$ A equipe multiprofissional de terapia intensiva da qual a enfermagem faz parte, necessita conhecer todos os aspectos da ME, para tratar o potencial doador de forma adequada. ${ }^{5}$

O presente estudo foi realizado com o objetivo de descrever a determinação da ME e a assistência de enfermagem na manutenção do potencial doador de órgãos.

\section{OBJETIVO}

Descrever a determinação da ME e a assistência de enfermagem 
na manutenção do potencial doador de órgãos, através de uma abordagem teórica sobre o assunto.

\section{MÉTODOS}

Trata-se de pesquisa bibliográfica realizada através de revisão de literatura científica sobre a determinação de ME e a assistência de enfermagem na manutenção do potencial doador.

Foram selecionados estudos e artigos através de busca eletrônica em base de dados MEDLINE/PUBMED (National Library of Medicine, USA), EMBASE (européia) e LILACS (Literatura Latino-Americana e do Caribe em Ciências da Saúde).

Não houve restrição com relação ao idioma ou à data de publicação. A seleção das publicações foi restrita aos estudos relacionados à determinação de ME, aos cuidados ao potencial doador de órgãos e à assistência de enfermagem na manutenção do potencial doador.

Outros estudos como manuais e livros foram incluídos no estudo, quando considerados relevantes.

Os descritores utilizados para a busca foram: transplantes, doação de órgãos, morte encefálica (ME), manutenção do potencial doador, cuidados de enfermagem e assistência de enfermagem.

O levantamento bibliográfico foi realizado no período de janeiro a setembro de 2009. Foram incluídos artigos que preenchiam os objetivos do estudo, sendo analisados em seu contexto e interpretados para confecção deste artigo.

O texto foi construído com subtítulos sobre determinação de $\mathrm{ME} \mathrm{e}$ assistência de enfermagem ao potencial doador de órgãos e tecidos para transplante, sendo este último apresentado na forma de quadro, incluindo as principais alterações, justificativas e cuidados a serem observados pela enfermagem.

\section{RESULTADOS E DISCUSSÃO}

Ao todo, foram selecionados 12 artigos, duas resoluções do Conselho Federal de Medicina e o Manual de Transplantes do Ministério da Saúde.

\section{Determinação de ME}

Para o diagnóstico de morte encefálica é essencial o cumprimento de pré-requisitos básicos, tais como: comprovação de lesão encefálica catastrófica e irreversível, ausência de distúrbios hidroeletrolíticos e ácido-base graves, intoxicação exógena, hipotermia ou ação de bloqueadores neuromusculares, benzodiazepínicos e outros sedativos. ${ }^{6}$ Diabetes insipidus com consequente hipernatremia ocorre em até $95 \%$ dos pacientes em morte encefálica. O diagnóstico de morte encefálica com teste de apnéia pode ser feito em vigência de hipernatremia, desde que a neuroimagem evidencie uma lesão incompatível com vida ou o teste complementar seja positivo. ${ }^{7}$

O exame clínico baseia-se na demonstração de coma profundo e irreversível (Glasgow 3), ausência de reflexos do tronco encefálico e presença de apnéia. ${ }^{8}$ Os reflexos de tronco testados incluem o fotomotor (resposta pupilar à luz), córneo-palpebral (estimulação da córnea com gaze ou algodão), oculocefálico (manobra dos olhos de boneca), oculovestibular (injeção de água gelada no conduto auditivo externo) e reflexo da tosse. ${ }^{1}$ Achados não neurológicos comuns em pacientes com morte encefálica incluem: perda da flutuação normal da frequência cardíaca (tipicamente fixa em uma determinada frequência), choque circulatório necessitando reposição volêmica e uso de drogas vasoativas, poliúria secundária a diabetes insipidus, coagulação intravascular disseminada, edema agudo de pulmão e acidose metabólica. ${ }^{8}$

A apnéia deve ser confirmada através do teste de apnéia, onde movimentos respiratórios espontâneos devem estar ausentes, com elevação adequada da $\mathrm{pCO}_{2} \cdot{ }^{9}$ Os pré-requisitos para realização do teste de apnéia incluem: temperatura $>36,5$, pressão sistólica $>$ $90 \mathrm{mmHg}$, balanço hídrico positivo, $\mathrm{pO}_{2}>200$ mmHg (mantendo $\mathrm{FiO}_{2}$ em $100 \%$, por pelo menos 10 minutos antes do início do teste). Se todos esses requisitos forem preenchidos, colhe-se uma gasometria arterial, retira-se o paciente da ventilação mecânica e coloca-se um cateter de oxigênio na cânula traqueal, com fluxo de seis a oito litros por minuto. Observa-se então o paciente por 10 minutos à procura de qualquer movimento respiratório. $\mathrm{O}$ teste de apnéia deve ser interrompido caso haja queda da saturação de O2 <89\% ou instabilidade hemodinâmica do paciente (pressão sistólica $<70 \mathrm{mmHg}$ ). Caso ocorram movimentos respiratórios, o teste deve ser interrompido imediatamente e o paciente conectado novamente ao respirador; ele não está em morte encefálica. Caso não haja movimento respiratório após 10 minutos, uma nova gasometria deve ser colhida e o paciente novamente conectado ao respirador. O teste de apnéia é considerado positivo se o paciente não apresentar movimento respiratório e a $\mathrm{pCO}_{2}$ no final do teste foi $>55 \mathrm{mmHg}$ ou subiu $>20 \mathrm{mmHg}$ do valor basal anterior ao teste. O teste é considerado negativo se houver qualquer movimento respiratório durante o procedimento. O teste de apnéia é considerado inconclusivo se não houver movimento respiratório durante o teste, porém se os níveis esperados de $\mathrm{pCO}_{2}$ na gasometria não forem atingidos. Alguns autores sugerem que o teste de apnéia seja realizado apenas depois que o teste confirmatório comprovar ausência de fluxo sanguíneo cerebral, pois a realização do mesmo em paciente ainda com circulação cerebral pode ser fatal. ${ }^{7}$ Eles também sugerem iniciar o teste com pCO $_{2} 40$ e 45 mmHg, podendo, portanto, reduzir o tempo de observação em apnéia para seis minutos, pois a pCO2 sobe em média $3 \mathrm{mmHg}$ por minuto durante a apnéia, consequentemente reduzindo as complicações (arritmias, acidose metabólica, hipocalemia, hipotensão).

Em adultos, se o exame clínico for compatível com morte encefálica, um segundo exame deve ser realizado após seis horas, geralmente por um segundo médico. Recomenda-se que pelo menos um dos exames seja feito por neurologista, neuropediatra ou neurocirurgião. No segundo exame clínico não há necessidade da realização do teste de apnéia. Crianças apresentam critérios de tempo entre exames diferentes de adultos: (a) é impossível obter diagnóstico de certeza com menos de sete dias; (b) entre sete dias e dois meses: 48 horas; (c) entre dois meses e um ano: 24 horas; (d) entre um e dois anos: 12 horas; (e) acima de dois anos: seis horas. ${ }^{9}$

A legislação brasileira obriga a realização de pelo menos um exame complementar confirmatório de morte encefálica. Os exames aceitos pelo Conselho Federal de Medicina como confirmatórios de morte encefálica são: angiografia cerebral (padrão-ouro), doppler transcraniano, eletroencefalograma e cintilografia cerebral. ${ }^{2}$ Embora sejam utilizados em alguns países, potenciais evocados somatossensitivos, angiotomografia e angioressonância de crânio não são exames reconhecidos pelo CFM para confirmação diagnóstica de ME no Brasil. 


\section{Assistência de Enfermagem ao potencial doador de órgãos e tecidos para transplante}

O potencial doador engloba todo paciente com lesão encefálica grave em coma Glasgow 3 de causa conhecida e compatível com o quadro clínico, em indivíduos sem contra-indicações para determinação de ME, a fim de manter os órgãos viáveis para possível transplante. Durante todo esse processo, a participação familiar deve ser incentivada para entendimento de todas as fases do processo e do conceito de irreversibilidade do quadro de ME. ${ }^{10}$

Nesse contexto, em Unidades de Emergência e Terapia Intensiva, todo paciente grave, mesmo depois da suspeição de $\mathrm{ME}$, deve ser tratado com a mesma seriedade e engajamento por toda a equipe, pois negligências nesse período poderão acarretar disfunção de órgãos no paciente potencial doador, com contra-indicação ou impedimentos ao aproveitamento dos órgãos passíveis de doação. $\mathrm{O}$ enfermeiro, como profissional envolvido diretamente no atendimento aos pacientes, deve auxiliar nos cuidados de potenciais doadores, ${ }^{10}$ evitando perda de órgãos por parada cardíaca durante o processo de determinação de ME.

Cabe à equipe de enfermagem auxiliar nos cuidados dos pacientes potenciais doadores, e, para tanto, o enfermeiro deve possuir domínio de todas as situações clínicas que podem ocorrer em decorrência da ME. ${ }^{3}$

A Figura 1 descreve as principais situações clínicas que ocorrem na ME, suas justificativas e cuidados a serem seguidos pela enfermagem..$^{5,11}$

Figura(s) 1. Principais complicações clínicas na ME, justificativas e cuidados de enfermagem.

\begin{tabular}{|c|c|c|}
\hline \multicolumn{3}{|c|}{ CARDIOVASCULARES } \\
\hline ALTERAÇÕES & JUSTIFICATIVA & CUIDADOS \\
\hline Hipertensão & $\begin{array}{l}\text { Ocorre geralmente } \\
\text { nas primeiras horas da } \\
\mathrm{ME} \text {, devido à descarga } \\
\text { de catecolaminas, } \\
\text { hipertensão intracraniana } \\
\text { (HIC) e Isquemia } \\
\text { - tratamento com } \\
\text { nitroprussiato de sódio. }\end{array}$ & $\begin{array}{l}\text { - Controle rigoroso } \\
\text { da pressão arterial } \\
\text { (PA). Administração } \\
\text { de nitroprussiato sob } \\
\text { prescrição médica com } \\
\text { controle rigoroso do } \\
\text { gotejamento ou utilização } \\
\text { de bomba de infusão } \\
\text { contínua. }\end{array}$ \\
\hline Hipotensão & $\begin{array}{l}\text { Ocorre devido a causas } \\
\text { prévias ao dano } \\
\text { cerebral: desidratação, } \\
\text { hipovolemia, diurese } \\
\text { osmótica, hipertermia } \\
\text { e lesão do centro } \\
\text { vasomotor: redução da } \\
\text { temperatura, depressão } \\
\text { da função miocárdica. }\end{array}$ & $\begin{array}{l}\text { - Manutenção da PA } \\
\text { sistólica >100 mmHg. } \\
\text { - Reposição de volume } \\
\text { cpm. } \\
\text { - Controle da pressão } \\
\text { venosa central (PVC) (>10 } \\
\text { cmH20). } \\
\text { - Administração de droga } \\
\text { vasoativa cpm. } \\
\text { - Administração de } \\
\text { hemocomponentes } \\
\text { cpm para manter a } \\
\text { hemoglobina }>10 \mathrm{~g} / \mathrm{dl} \text {. }\end{array}$ \\
\hline & & \\
\hline
\end{tabular}

\begin{tabular}{|c|c|c|}
\hline Arritmias & $\begin{array}{l}\text { Alterações mais comuns: } \\
\text { alterações segmento } \\
\text { ST, onda T invertida, } \\
\text { bloqueio atrioventricular e } \\
\text { bradiarritmias. } \\
\text { Ocorrem pela falta do } \\
\text { estímulo simpático ao } \\
\text { coração e perda da } \\
\text { função do tronco cerebral. } \\
\text { Tais deficiências } \\
\text { de condução são } \\
\text { secundárias à } \\
\text { hipovolemia, hipóxia e } \\
\text { isquemia miocárdica. }\end{array}$ & $\begin{array}{l}\text { - Monitorização cardíaca. } \\
\text { - Providenciar material } \\
\text { para implante de } \\
\text { marcapasso externo, } \\
\text { quando necessário. } \\
\text { - Manter suporte } \\
\text { hidroeletrolítico. } \\
\text { - Controle rigoroso das } \\
\text { alterações nos exames } \\
\text { laboratoriais. }\end{array}$ \\
\hline Taquicardia & $\begin{array}{l}\text { Ocorre devido à descarga } \\
\text { de catecolaminas. }\end{array}$ & $\begin{array}{l}\text { - Manter controle rigoroso } \\
\text { de sinais vitais. } \\
\text { - Monitorização cardíaca. }\end{array}$ \\
\hline
\end{tabular}

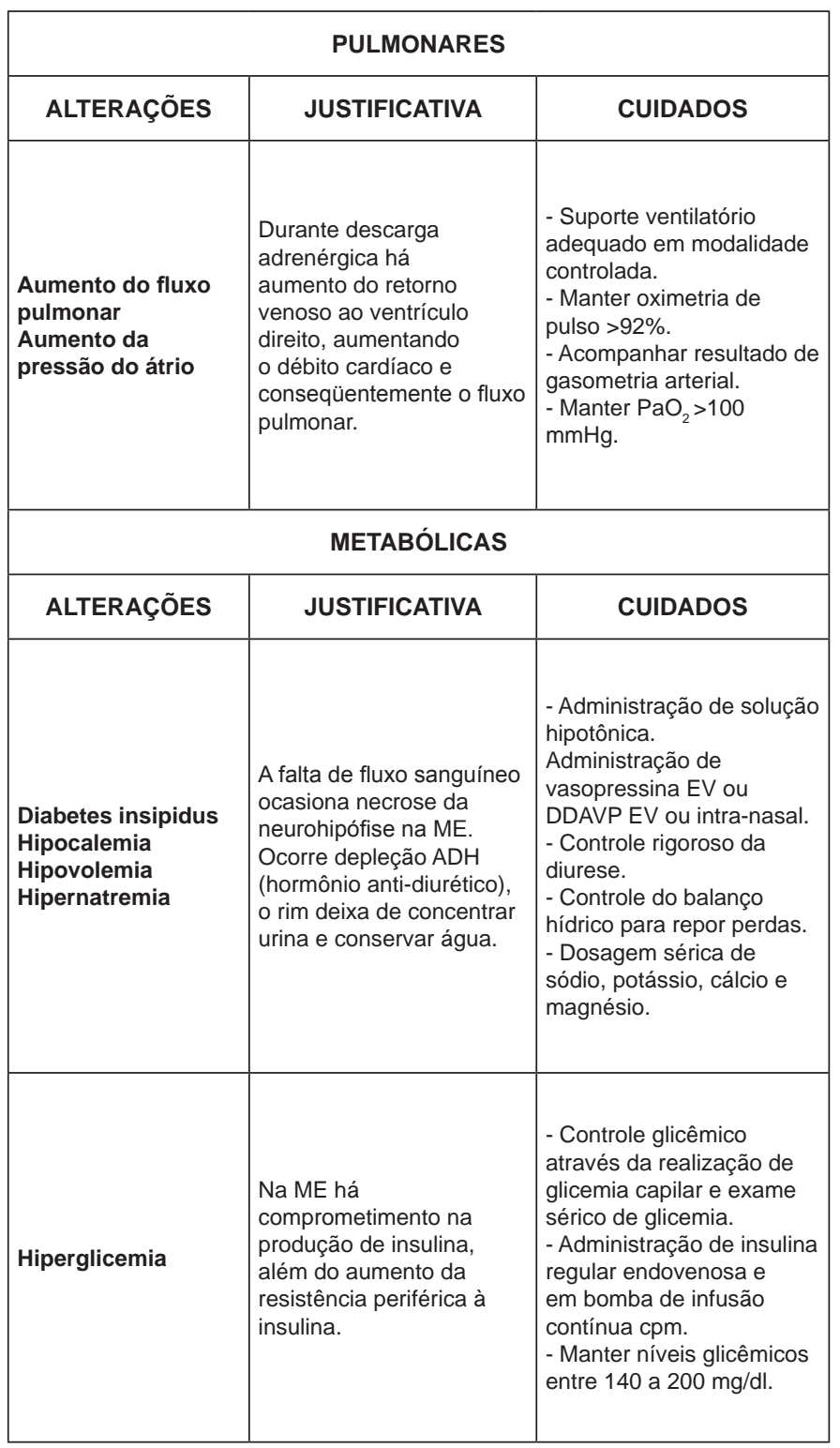




\begin{tabular}{|c|l|l|}
\hline \multicolumn{3}{|c|}{ ALTERAÇÕES DA TEMPERATURA CORPORAL } \\
\hline ALTERAÇÕES & \multicolumn{1}{|c|}{ JUSTIFICATIVA } & \multicolumn{1}{c|}{ CUIDADOS } \\
\hline \multirow{3}{*}{ Hipotermia } & & $\begin{array}{l}\text { - Controle rigoroso de } \\
\text { temperatura (manter } \\
\left.>35^{\circ} \mathrm{C}\right) .\end{array}$ \\
& $\begin{array}{l}\text { - Utilizar medidas de } \\
\text { reaquecimento: infusão } \\
\text { de soluções aquecidas } \\
\text { baixas podem provocar } \\
\text { arritmias cardíacas, } \\
\text { hipocontratilidade } \\
\text { cardíaca, alterações na } \\
\text { coagulação e na função } \\
\text { gástricas e vesicais com } \\
\text { soro aquecido, uso de } \\
\text { cobertores térmicos e } \\
\text { nebulização aquecida. }\end{array}$ \\
\hline
\end{tabular}

\begin{tabular}{|c|c|c|}
\hline \multicolumn{3}{|c|}{ RENAIS } \\
\hline ALTERAÇÕES & JUSTIFICATIVA & CUIDADOS \\
\hline $\begin{array}{l}\text { Baixo débito } \\
\text { urinário. } \\
\text { Poliúria. }\end{array}$ & $\begin{array}{l}\text { Utilizar sonda vesical de } \\
\text { demora para controle } \\
\text { rigoroso da diurese } \\
\text { horária. }\end{array}$ & $\begin{array}{l}\text { - Manter débito urinário em } \\
\text { torno de } 1 \mathrm{ml} / \mathrm{Kg} / \text { hora no } \\
\text { adulto e } 2 \mathrm{ml} / \mathrm{Kg} / \mathrm{hora} \text { na } \\
\text { criança. } \\
\text { - Avaliar hipovolemia, } \\
\text { hipotensão, obstrução ou } \\
\text { vazamento da sonda. } \\
\text { - Acompanhar dosagem } \\
\text { sérica de creatinina, para } \\
\text { avaliação da função renal. }\end{array}$ \\
\hline \multicolumn{3}{|c|}{ INFECCIOSAS } \\
\hline ALTERAÇÕES & JUSTIFICATIVA & CUIDADOS \\
\hline $\begin{array}{l}\text { HIGIENIZAÇÃO } \\
\text { Corporal e oral }\end{array}$ & $\begin{array}{l}\text { Manter a higiene visando } \\
\text { a boa aparência do } \\
\text { paciente, diminuir a } \\
\text { impressão de descuido e } \\
\text { prevenir infecções. } \\
\text { Pode ocorrer } \\
\text { ressecamento, que pode } \\
\text { ocasionar opacificação } \\
\text { das córneas, além do } \\
\text { acúmulo de secreções, } \\
\text { predispondo à infeç̧̃̃es. }\end{array}$ & $\begin{array}{l}\text { Realizar higiene corporal } \\
\text { e oral conforme rotina do } \\
\text { setor. } \\
\text { - Manter olhos umedecidos } \\
\text { com soro fisiológico. } \\
\text { - Utilização de pomada } \\
\text { oftálmica com freqüência } \\
\text { após higiene ocular, cpm. }\end{array}$ \\
\hline & & \\
\hline
\end{tabular}

\begin{tabular}{|l|l|l|}
\hline ALTERAÇÕES & \multicolumn{1}{|c|}{ JUSTIFICATIVA } & \multicolumn{1}{c|}{ CUIDADOS } \\
\hline Olhos & $\begin{array}{l}\text { Complicações infecciosas } \\
\text { devem ser evitadas. }\end{array}$ & $\begin{array}{l}\text { - Manter precauções } \\
\text { universais em todos os } \\
\text { procedimentos. } \\
\text { - Administração de } \\
\text { antibióticos cpm. }\end{array}$ \\
\hline
\end{tabular}

O reconhecimento precoce das principais complicações proporciona maior preservação dos órgãos.5

Em caso de parada cardiorrespiratória (PCR), devem ser iniciadas as manobras de reanimação, as quais devem promover uma imediata e adequada perfusão dos órgãos, o que não contraindicaria a provável doação.11

\section{CONCLUSÕES}

Todo processo de determinação de ME prolonga-se por várias horas até a confirmação do diagnóstico e definição sobre a doação pelos familiares do paciente.

A determinação da ME segue resoluções determinadas pelo Conselho Federal de Medicina, tendo caráter legal e objetivando definir o diagnóstico, determinar condutas médicas e/ou possibilitar a doação de órgãos e tecidos para fins de transplante.

Durante todo período de determinação, os cuidados médicos e de enfermagem devem ser continuados e direcionados para as principais complicações decorrentes do quadro de ME, visando a adequada manutenção dos órgãos e a viabilidade dos mesmos para transplante.

Trata-se de procedimento que envolve várias questões éticas relacionadas à sua determinação, abordagem e acompanhamento familiar, além de implicações legais em todo processo, seja na suspensão de medidas terapêuticas e de suporte quanto na captação de órgãos com a finalidade de doação.

Nesse contexto, o conhecimento por parte de toda equipe multiprofissional, entre elas o pessoal da enfermagem torna-se um diferencial necessário ao sucesso do objetivo final na doação.

Este estudo forneceu informações relacionadas à ME, questões éticas a ela relacionadas e à assistência de enfermagem nesses casos, visando melhorar e buscar excelência nos cuidados a esses pacientes.

\section{ABSTRACT}

Brain death means the irreversible end of every brain activity, and when it is determined following standard protocols, it poses the optimal opportunity to the organs donation, whenever there is the family members' consent. The adequate patient's hemodynamic maintenance performed both by the physician and the nursing staff is of utmost importance to the organs feasibility. Purposes: To describe the brain death determination criteria, as well as the standards to be used by the nursing care in keeping the organs feasibility. Methods: it was performed a search in the literature among the main international indexed database, looking for articles, resolutions and books covering such issue. Results: All patients with severe brain injury of known cause, Glasgow 3 coma, no brainstem reflexes, and apnea in two different clinical examinations and positive complementary test are potential organ donors. The nursing staff should be aware on the possible clinical complications that could damage the organs feasibility in brain death patients before these organs can be surgically removed. Conclusions: The brain death determination has ethical and legal aspects as to the organ donation. The maintenance of the organs feasibility of potential donors should be one of the main objectives in the medical care.

Keywords: Brain Death, Nursing Care, Transplants. 


\section{REFERÊNCIAS}

1. Wijdicks EFM. Brain Death Worldwide: Accepted Fact but no Global Consensus in Diagnostic Criteria. Neurology. 2002;58: 20-25.

2. Conselho Federal de Medicina. Resolução. CFM nº 1.480/97 de 08 de agosto de 1997. Diário Oficial da União em 21 de agosto de 1997. Página 18227.

3. Araujo S, Cintra EA, Bachega EB. Manutenção do potencial doador de órgãos. In: Cintra EA, Nishide VM. Nunes WA. São Paulo: Atheneu; 2005.

4. Rech TH, Rodrigues Filho EM. Manuseio do Potencial Doador de Múltiplos Órgãos. Rev Bras Ter Int. 2007;19:197-204.

5. Guetti NR, MARQUES IR. Assistência de enfermagem ao potencial doador de órgãos em morte encefálica. Rev Bras Enferm. Brasília. 2008;61(1):91-7.

6. Wijdicks EFM. The Diagnosis of Brain Death. New Eng J Med. 2001;344:1215-21.
7. Andrade AF, Amorim RLO, Paiva WS, Figueiredo EG, Barros e Silva LB, Teixeira MJ. Proposta para revisão dos critérios clínicos de morte encefálica. Rev Med. 2007;86:132-37.

8. American Academy of Neurology Guidelines on the Determination of Brain Death. Neurology. 1995;45:1012-4.

9. Andrade AF, Paiva WS, Amorim RLO, Figueiredo EG, Barros e Silva LB, Teixeira MJ. O teste de apnéia no diagnóstico de morte encefálica. Rev Med. 2007;86:138-43.

10. Ministério da Saúde (BR). Secretaria do Estado de Saúde do Paraná. Manual de Transplantes. 2.ed; 2004.

11. Bachega EB, Hilário N, Oliveira. Lúcia MR. Manual de Enfermagem em Urologia. Captação de Órgãos: Papel do Enfermeiro. Campinas: UNICAMP, 2001. p. 51-62. 